\title{
Vacuum arc plasma generation and thin film deposition from a TiB2 cathode
}

\author{
Igor Zhirkov, Andrejs Petruhins, Lars-Åke Näslund, Szilard Kolozsvari, Peter Polcik and \\ Johanna Rosén
}

\section{Linköping University Post Print}

\section{Tweet}

N.B.: When citing this work, cite the original article.

Original Publication:

Igor Zhirkov, Andrejs Petruhins, Lars-Åke Näslund, Szilard Kolozsvari, Peter Polcik and Johanna Rosén, Vacuum arc plasma generation and thin film deposition from a TiB2 cathode, 2015, Applied Physics Letters, (107), 18, 184103.

http://dx.doi.org/10.1063/1.4935152

Copyright: American Institute of Physics (AIP)

http://www.aip.org/

Postprint available at: Linköping University Electronic Press

http://urn.kb.se/resolve?urn=urn:nbn:se:liu:diva-123139 


\section{A|P |Applied Physics \\ Letters}

\section{Vacuum arc plasma generation and thin film deposition from a TiB2 cathode}

Igor Zhirkov, Andrejs Petruhins, Lars-Ake Naslund, Szilard Kolozsvári, Peter Polcik, and Johanna Rosen

Citation: Applied Physics Letters 107, 184103 (2015); doi: 10.1063/1.4935152

View online: http://dx.doi.org/10.1063/1.4935152

View Table of Contents: http://scitation.aip.org/content/aip/journal/apl/107/18?ver=pdfcov

Published by the AIP Publishing

\section{Articles you may be interested in}

Cathodic cage plasma deposition of TiN and $\mathrm{TiO} 2$ thin films on silicon substrates

J. Vac. Sci. Technol. A 33, 041502 (2015); 10.1116/1.4919770

Effect of Ti-Al cathode composition on plasma generation and plasma transport in direct current vacuum arc J. Appl. Phys. 115, 123301 (2014); 10.1063/1.4869199

Preparation and characterization of high-quality TiN films at low temperature by filtered cathode arc plasma J. Vac. Sci. Technol. A 22, 2419 (2004); 10.1116/1.1807836

Erratum: "Raman spectroscopy and x-ray diffraction studies of (Ti,Al)N films deposited by filtered cathodic vacuum arc at room temperature" [J. Appl. Phys. 89, 6192 (2001)]

J. Appl. Phys. 91, 10231 (2002); 10.1063/1.1483106

Raman spectroscopy and x-ray diffraction studies of (Ti,Al)N films deposited by filtered cathodic vacuum arc at room temperature

J. Appl. Phys. 89, 6192 (2001); 10.1063/1.1352564

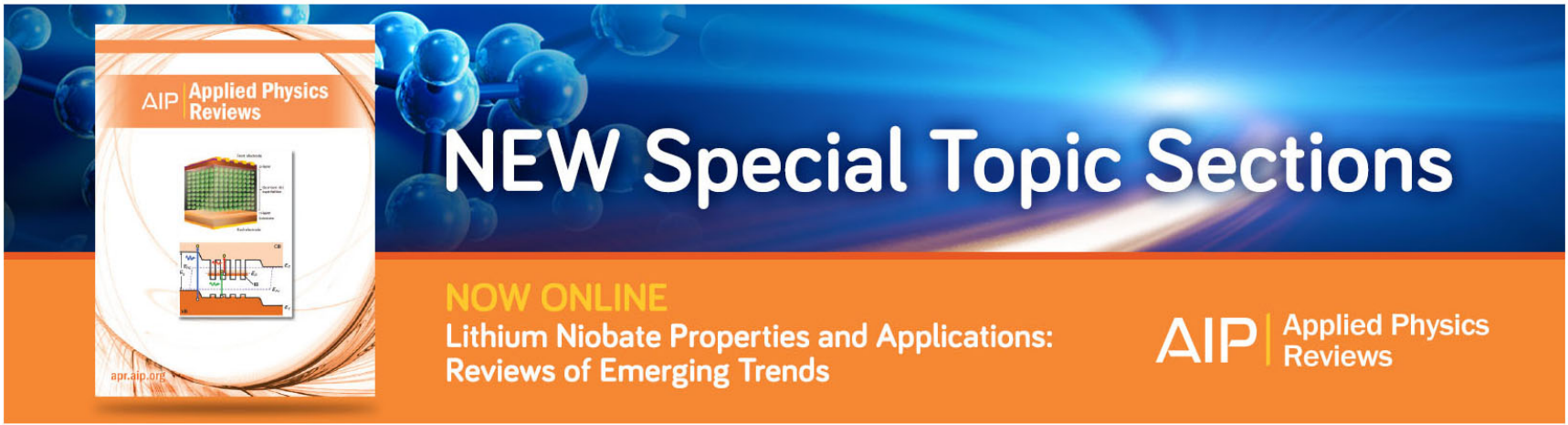




\title{
Vacuum arc plasma generation and thin film deposition from a $\mathrm{TiB}_{2}$ cathode
}

\author{
Igor Zhirkov, ${ }^{1, a)}$ Andrejs Petruhins, ${ }^{1}$ Lars-Ake Naslund, ${ }^{1}$ Szilard Kolozsvári, ${ }^{2}$ Peter Polcik, ${ }^{2}$ \\ and Johanna Rosen ${ }^{1}$ \\ ${ }^{1}$ Thin Film Physics Division, Department of Physics, Chemistry and Biology (IFM), Linköping University, \\ SE-581 83 Linköping, Sweden \\ ${ }^{2}$ PLANSEE Composite Materials GmbH, Siebenbürgerstraße 23, 86983 Lechbruck am See, Germany
}

(Received 12 August 2015; accepted 22 October 2015; published online 4 November 2015)

\begin{abstract}
We have studied the utilization of $\mathrm{TiB}_{2}$ cathodes for thin film deposition in a $\mathrm{DC}$ vacuum arc system. We present a route for attaining a stable, reproducible, and fully ionized plasma flux of Ti and B by removal of the external magnetic field, which leads to dissipation of the vacuum arc discharge and an increased active surface area of the cathode. Applying a magnetic field resulted in instability and cracking, consistent with the previous reports. Plasma analysis shows average energies of 115 and $26 \mathrm{eV}$, average ion charge states of 2.1 and 1.1 for Ti and $\mathrm{B}$, respectively, and a plasma ion composition of approximately $50 \% \mathrm{Ti}$ and $50 \% \mathrm{~B}$. This is consistent with measured resulting film composition from X-ray photoelectron spectroscopy, suggesting a negligible contribution of neutrals and macroparticles to the film growth. Also, despite the observations of macroparticle generation, the film surface is very smooth. These results are of importance for the utilization of cathodic arc as a method for synthesis of metal borides. (C) 2015 AIP Publishing LLC.

[http://dx.doi.org/10.1063/1.4935152]
\end{abstract}

A large subgroup of borides is the metal diborides, in particular, those with the hexagonal $\mathrm{AlB}_{2}$ type structure, in which the $\mathrm{B}$ atoms form strongly bonded graphene-like sheets between layers of metal atoms. The most studied diborides include the transition metal diborides $\mathrm{TiB}_{2}, \mathrm{ZrB}_{2}$, $\mathrm{MgB}_{2}$, and $\mathrm{HfB}_{2}$, with high hardness, chemical and thermal stability, as well as good electrical conductivity. ${ }^{1-4}$ This unique property envelope for ultrahigh temperature ceramics renders them suitable for extreme applications including space, cutting tools, engines, etc. In particular, $\mathrm{TiB}_{2}$ is crucial for machining aluminum, ubiquitous in the automobile, aerospace, and telecommunications industries. Deposition of $\mathrm{TiB}_{2+\mathrm{x}}$ coatings, typically columnar $\mathrm{TiB}_{2}$ with a B-rich tissue phase, by physical vapor deposition (PVD) has been previously demonstrated by magnetron sputtering from compound targets. ${ }^{5-9}$ However, amongst PVD techniques, arc evaporation is more commonly used for synthesis of hard coatings, mainly because of high deposition rate, good adhesion, and a dense morphology. ${ }^{10}$ Till date, there is no reproducible synthesis of $\mathrm{TiB}_{2 \pm \mathrm{x}}$ from arc evaporation and from the reported few attempts on the use of $\mathrm{TiB}_{2}$ cathodes for thin film synthesis, extensive instability, cracking, and cathode failure. ${ }^{11}$ Hypothetical routes for improving the process stability has been suggested as follows: adding carbon into the cathode, applying a "suitable" magnetic field, or working in a reactive atmosphere, ${ }^{11}$ or to simply use pulsed arc at the expense of, e.g., a high deposition rate. ${ }^{12}$ These routes remain to be confirmed, although a stable arc process has been reported for the use of a $\mathrm{TiB}_{2}$ cathode in $\mathrm{N}_{2}$ for synthesis of nitride multielement alloys. ${ }^{13}$

In this letter, we have explored conditions allowing the stable vacuum arc plasma generation from a $\mathrm{TiB}_{2}$ cathode. The erosion of the cathode surface is correlated to

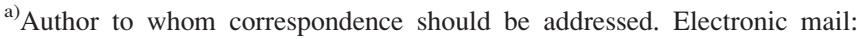
igozh@ifm.liu.se. Tel.: +46 7305210 12. Fax: +46 13137568.
}

charge-state-resolved plasma analysis of ion chemistry and ion energy, macroparticle generation, as well as resulting film composition. We show that the absence of a magnetic field prevent cracking of the cathode and allow stable and reproducible plasma generation and film formation in vacuum. These results are of high importance for providing a path for controlled materials' synthesis from $\mathrm{TiB}_{2}$ cathodes in vacuum arc and an increased fundamental understanding of the plasma generation from metal boride cathodes.

The experiments were performed using a deposition system equipped with an industrial scale DC arc plasma source (Ionbond) with cathodes with a diameter of $63 \mathrm{~mm}$. In all experiments, the arc source was operated at $65 \mathrm{~A}$ arc current at the system base pressure around $10^{-4} \mathrm{~Pa}\left(\sim 10^{-6}\right.$ Torr $)$. The $\mathrm{TiB}_{2}$ cathodes were provided by PLANSEE Composite Materials and equipped with a protective stainless steel ring for the event of possible cathode destruction. Instead of a permanent ring magnet, which usually serves to keep the arc spot on the working surface of the cathode, the influence of the magnetic field on the process stability was controlled through a coil of the same geometry and in the same position as a ring magnet in a typical scheme of an arc source.

Figs. 1(a) and 1(b) show the cathode surface during plasma generation in the absence and in the presence of a magnetic field, respectively, in both cases accompanied by the emission of glowing particles.

In the absence of a magnetic field, see Fig. 1(a), the arc is homogeneously distributed over the active part of the cathode surface. Attempts were performed to apply an external magnetic field; however, even a weak field $(<100 \mu \mathrm{T})$ leads to collapse of the active region into a much smaller cluster (see Fig. 1(b)), which tends to stick to its location at the cathode surface leading to local overheating with initiated cracking. The latter is consistent with the previous observations. ${ }^{11}$ The influence of the magnetic field on the dissipation of the arc on a boride surface needs to be further explored, though 

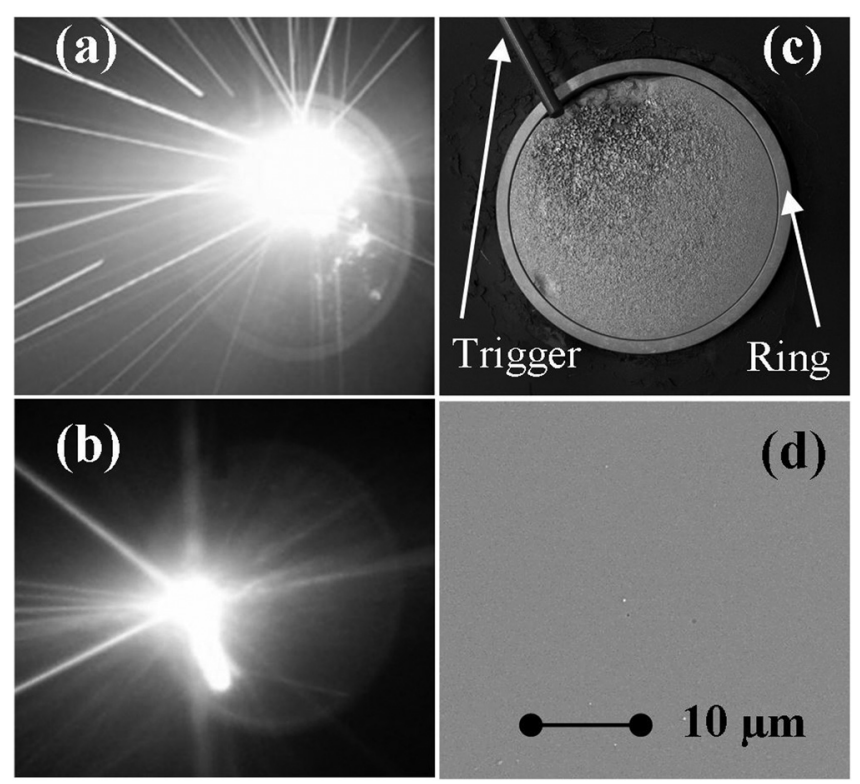

FIG. 1. Photos (exposure time 1/100 s) of the cathode surface during plasma generation in (a) the absence and (b) the presence of a magnetic field. (c) The cathode surface after $10 \mathrm{~min}$ of arcing. (d) SEM diagnostics of the deposited film on a $\mathrm{MgO}$ substrate.

these first results indicate that magnetic field (or absence thereof) is a route for process stabilization, allowed by advancing beyond traditional permanent ring magnets of the arc source.

Fig. 1(c) shows the surface of the cathode after $10 \mathrm{~min}$ of plasma generation. Clear traces of erosion are evident on more than $50 \%$ of the surface. An increased surface roughness closer to the trigger indicates a more frequently operated region of the surface. Initial cracking and lost pieces of the cathode can also be seen close to the protective ring. However, this is a result of the initial attempts to apply a magnetic field and likely also enhanced by the triggering scheme of the source. During arc ignition, when the trigger pin is connected to the cathode surface, all current flows through a relatively small area. In the presence of a field, dissipation of the arc spot is reduced (as shown in Fig. 1(b)) and hence overheating close to the trigger can be expected.

Scanning electron microscopy (SEM) on the virgin cathode surface (top view), see Fig. 2(a), shows grains below $10 \mu \mathrm{m}$. After plasma generation, see Fig. 2(b), the crystalline features are of a reduced size and accompanied by a smooth surface layer, with apparent cracks and being partly detached from the body of the cathode. The latter may be explained by molten regions forming on the surface during arcing and a thermal expansion coefficient of $\mathrm{TiB}_{2}$ causing separation of the regions being molten/solid. ${ }^{2}$ It should be noted that this coefficient has previously been used to explain failing cathodes for non-dissipating arc spots.

It is known that sharp crystal grains/edges lead to an enhancement of electron emission and promote initiation of the arc spot. This can be compared to arcing from poisoned cathodes, where dissipation of the arc spot occurs due to charging of contaminations under ion bombardment, creating an electric field which enhances electron emission. ${ }^{10}$ The rate of charging and the field enhancement and emission onset are very fast and several emission centers switch on

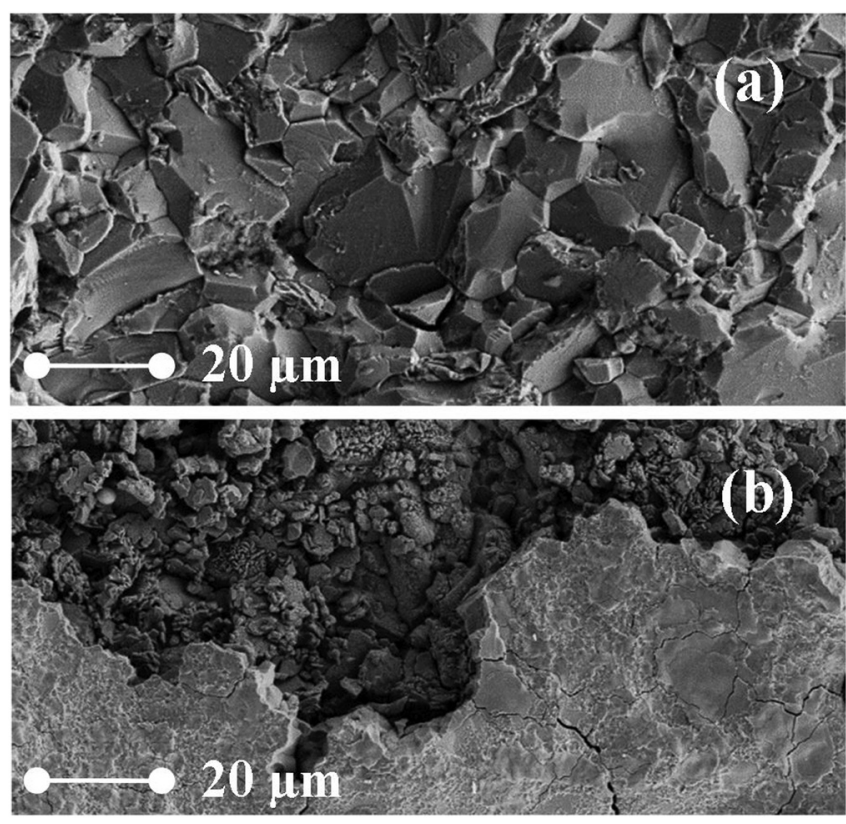

FIG. 2. SEM micrograph of the (a) virgin and (b) used cathode surface.

simultaneously. ${ }^{14,15}$ Since the crystalline structure of $\mathrm{TiB}_{2}$ is clearly seen even on the surface of the used cathode, arc dissipation through spot ignition on the edges of the crystals is suggested. The dissipation results in a reduced current per spot and hence a larger area subjected to heating by the arc current. The latter allows a reduction of local heating of the cathode and therefore a stabilized DC vacuum arc.

The possibility of dissipation of the arc discharge due to the edges of the cathode crystals requires their presence also after plasma generation. It can be assumed that after spot ignition, some of the cathode material will be melted and a part of the surface smoothened. As shown in Fig. 2(b), the smoothened thin layer is weakly attached to the cathode body. Because of the explosive nature of the arc, it is possible that the arc continuously destroys the solidified smoothened layer, ${ }^{16}$ allowing the crystals beneath the surface. However, this process does not influence the film growth, which is evident from the deposited films showing a very smooth surface (see Fig. 1(d)).

The ionized flux from the $\mathrm{TiB}_{2}$ cathode was characterized with a mass-energy-analyzer (MEA, Hiden Analytics model EQP) through measurements of charge-state-resolved plasma chemistry and ion energy distributions (IEDs). The analyzer orifice $(50 \mu \mathrm{m}$ diameter) was placed at the same position as the substrate holder. The plasma was generated with no external magnetic field on the cathode surface, and mass-scans at fixed ion energy and energy-scans at fixed mass-to-charge ratio were acquired for all detected ions. The energy scans were recorded in steps of $0.25 \mathrm{eV} /$ charge up to $200 \mathrm{eV} /$ charge to capture the entire IED. The presence of isotopes in the ion flux and their influence on the relative ratios of the measured IEDs were evaluated according to the previous work. ${ }^{17}$ Each IED was recorded at least three times to ensure consistency of the data. The results of the diagnostic are presented in Fig. 3.

The highest ion charge state detected is $4+$ for both $\mathrm{Ti}$ and $\mathrm{B}$, where $\mathrm{Ti}^{2+}$ and $\mathrm{B}^{1+}$ ions were found to be dominant. 


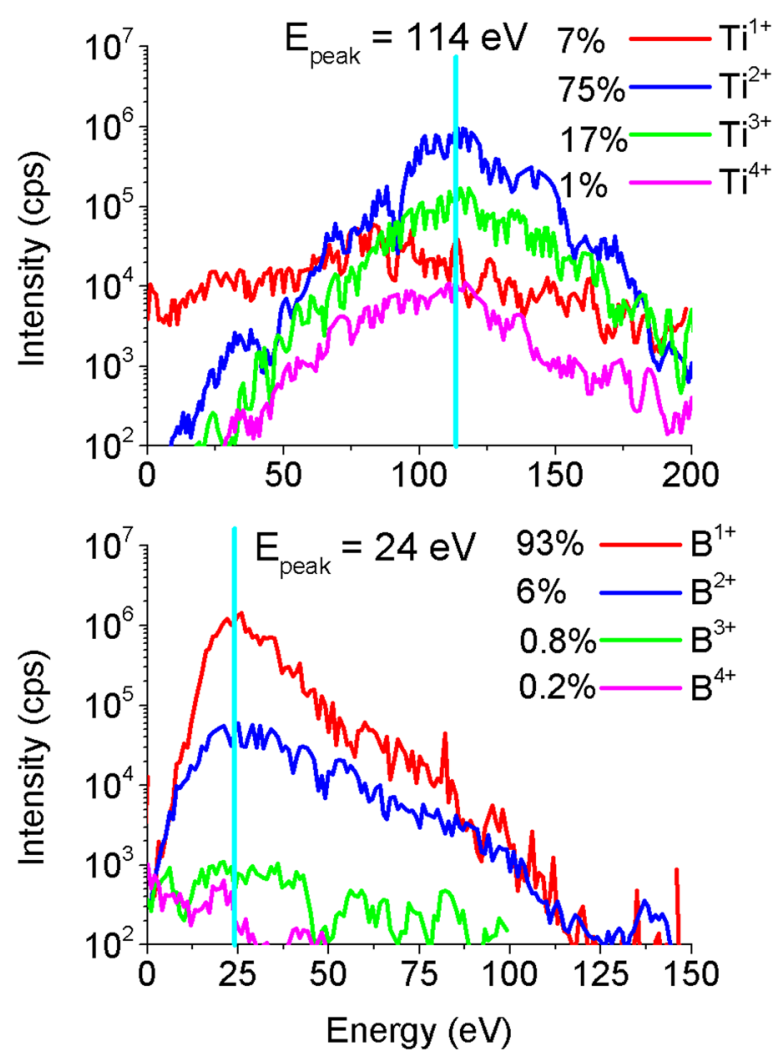

FIG. 3. IEDs of $\mathrm{Ti}$ and $\mathrm{B}$ ions from a $\mathrm{TiB}_{2}$ cathode operated at base pressure. The percentage of each ion species is obtained from integration of the corresponding IEDs.

The average charge states of $\mathrm{Ti}$ and $\mathrm{B}$ ions are 2.1 and 1.1, respectively, where the higher charge states of $\mathrm{Ti}$ originates from the lower ionization energies $\left(\mathrm{Ti}^{0} \rightarrow \mathrm{Ti}^{2+}=20.6 \mathrm{eV}\right.$; $\left.\mathrm{B}^{0} \rightarrow \mathrm{B}^{2+}=33.5 \mathrm{eV}\right)$. The IEDs extend up to $>200 \mathrm{eV}$ and $\sim 125 \mathrm{eV}$ for $\mathrm{Ti}$ and $\mathrm{B}$ ions, respectively, with corresponding average ion energies being $115 \mathrm{eV}$ and $26 \mathrm{eV}$. More energetic $\mathrm{Ti}$ ions can be explained by the recently suggested "velocity rule," 18 which states that the most-likely (peak) velocity of ions from a compound cathode in an arc discharge is the same. As a result, the different atomic mass of $\mathrm{Ti}$ and $\mathrm{B}$ (48 amu and $11 \mathrm{amu}$ ) determines the discrepancy between their peak kinetic energies (calculated Ti-114 eV and B$24 \mathrm{eV}$ ). To determine the total plasma ion composition, the integrated IEDs were summarized to a total of 56 at. $\% \mathrm{Ti}$ and 44 at. $\%$ B. This result is not consistent with the $\mathrm{TiB}_{2}$ cathode composition. However, the material flux from the cathode in an arc discharge is composed of ions, macroparticles, and neutrals, which may all contribute to the resulting film. While DC arc plasma is usually characterized by a small amount of neutrals, it is not expected to be significant for the here investigated plasma flux from a $\mathrm{TiB}_{2}$ cathode, with an evaporation temperature of $3215^{\circ} \mathrm{C}$.

To correlate between plasma generation and film formation, thin films were deposited on a grounded $\mathrm{MgO}$ substrate placed in the previous position of the plasma analyzer ( $33 \mathrm{~cm}$ from the cathode surface). It should be noted that despite the presence of glowing particles, see Fig. 1(a), the film surface is very smooth (see Fig. 1(d)). Analysis of film composition with X-ray photoelectron spectroscopy (XPS), calibrated with reference samples $\mathrm{TiB}_{2}$, metallic Ti, and pure $\mathrm{B}$, showed $53( \pm 2)$ at. $\%$ of $\mathrm{Ti}$ and $47( \pm 2)$ at. $\%$ of $\mathrm{B}$. The XPS results also confirm the $\mathrm{Ti}^{2+}$ oxidation state of the $\mathrm{TiB}$, as opposed to $\mathrm{Ti}^{4+}$ of $\mathrm{TiB}_{2}$. The smooth film surface together with the very similar composition of the plasma ions and the film suggests that the film originates from a close to fully ionized plasma, with a minor contribution from neutrals and macroparticles.

The discrepancy in composition between the cathode (33 at. $\%$ of Ti) and the plasma/film ( $\sim 50$ at. $\%$ of Ti) is considerable and may be explained by the previously shown dependence of spatial plasma distribution on ion mass. It is known that lighter elements typically have a wider distribution, ${ }^{19}$ and a reduced intensity along the cathode normal can be expected for lighter ions as compared to heavier elements. This is consistent with the here observed reduction of $\mathrm{B}$, compared with Ti, in both plasma and film.

Cathodic arc is a commonly used synthesis method for hard coating applications due to advantages such as a high deposition rate, good adhesion, and a close to fully ionized plasma flux. However, till date, this technique has not been utilized for deposition of $\mathrm{TiB}_{2 \pm \mathrm{x}}$ due to process instability and non-reproducible synthesis in the standard scheme of industrial arc sources. Here, we present a path for attaining a stable and fully ionized plasma flux of $\mathrm{Ti}$ and $\mathrm{B}$ elements with relatively high kinetic ion energy from a pure $\mathrm{TiB}_{2}$ cathode in vacuum, obtained by modifying the magnetic field and the traditional arc source scheme including a permanent magnet. It should be noted that the correlation between the plasma and the film composition is included in the present work to increase the fundamental understanding of the synthesis process. However, the measured discrepancy between cathode and film composition can be to a large extent compensated for by a focusing filter or by a nonstationary source-film setup, though that is beyond the scope of the present study. Altogether, the results are not only of importance for the utilization of cathodic arc as an efficient method for synthesis of metal borides but also for an increased understanding of the interplay between external experimental parameters and the composition and microstructure of the (compound) cathode.

The suggested stabilization of plasma generation through dissipation of the arc spots, which increase the active cathode surface area and reduce the heat load, can also be applicable in other arc processes. For instance, for the development of high current interrupters, the presence of high current arc spots and resulting non-uniform erosion of the electrodes are undesirable. From a fundamental point of view, further exploration of arc spot dissipation on crystal edges and the influence of a magnetic field will improve the understanding of arc plasma generation and potentially allow control of synthesis of new materials, of new compositions, by novel arc source as well as novel cathode design.

In conclusion, we have investigated the use of $\mathrm{TiB}_{2}$ cathodes for thin film deposition in a DC vacuum arc system. We show that the removal of an external magnetic field leads to dissipation of the vacuum arc discharge and an increased active area of the cathode, resulting in the prevention of overheating and a stabilization of the plasma generation. Plasma analysis shows average ion energies of 115 and $26 \mathrm{eV}$ for $\mathrm{Ti}$ and $\mathrm{B}$, respectively, and a plasma ion chemistry 
of approximately $50 \% \mathrm{Ti}$ and $50 \% \mathrm{~B}$. This is consistent with the measured resulting film composition. Also, despite the observations of macroparticle generation, the film surface is very smooth. These results are of importance for the utilization of cathodic arc as an efficient method for synthesis of metal borides.

The research was funded by the European Research Council under the European Community Seventh Framework Program (FP7/2007-2013)/ERC Grant Agreement No. 258509. J.R. acknowledges the funding from the Swedish Research Council (VR) Grant No. 642-2013-8020, from the Knut and Alice Wallenberg Foundation, and from the SSF synergy grant FUNCASE.

${ }^{1}$ S. A. Tucker and H. R. Moody, "The preparation of some new metal Borides," J. Chem. Soc. 81, 14-17 (1902).

${ }^{2}$ R. G. Munro, "Material properties of titanium diboride," J. Res. Natl. Inst. Stand. Technol. 105(5), 709-720 (2000).

${ }^{3}$ J. Nagamatsu, N. Nakagawa, T. Muranaka, Y. Zenitani, and J. Akimitsu, "Superconductivity at $39 \mathrm{~K}$ in magnesium diboride," Nature 410, 63-64 (2001).

${ }^{4}$ A. Passarone, F. Valenza, and M. Muolo, "A review of transition metal diborides: From wettability studies to joining," J. Mater. Sci. 47, 8275 (2012).

${ }^{5}$ C. Mitterer, "Borides in thin film technology," J. Solid State Chem. 133, 279-291 (1997).

${ }^{6} \mathrm{P}$. Losbichler and C. Mitterer, "Non-reactively sputtered TiN and TiB2 films: Influence of activation energy on film growth," Surf. Coat. Technol. 97(1-3), 567-573 (1997).

${ }^{7}$ M. Berger, L. Karlsson, M. Larsson, and S. Hogmark, "Low stress TiBcoatings with improved tribological properties," Thin Solid Films 401, 179-186 (2001).
${ }^{8}$ P. Mayrhofer, C. Mitterer, J. Wen, J. Greene, and I. Petrov, "Self-organized nanocolumnar structure in superhard $\mathrm{TiB}_{2}$ thin films," Appl. Phys. Lett. 86, 131909 (2005).

${ }^{9}$ F. Kunc, J. Musil, P. Mayrhofer, and C. Mitterer, "Low-stress superhard Ti-B films prepared by magnetron sputtering," Surf. Coat. Technol. 174-175, 744-753 (2003).

${ }^{10}$ A. Anders, Cathodic Arcs. From Fractal Spots to Energetic Condensation, 1st ed. (Springer, New York, 2008).

${ }^{11}$ O. Knotek, F. Löffler, M. Böhmer, R. Breidenbach, and C. Stöbel, "Ceramic cathodes for arc-physical vapour deposition: Development and application," Surf. Coat. Technol. 49, 263-267 (1991).

${ }^{12} \mathrm{~J}$. Treglio, S. Trujillo, and A. Perry, "Deposition of $\mathrm{TiB}_{2}$ at low temperature with low residual stress by a vacuum arc plasma source," Surf. Coat. Technol. 61, 315-319 (1993).

${ }^{13}$ H. Fager, J. Andersson, J. Jensen, J. Lu, and L. Hultman, "Thermal stability and mechanical properties of amorphous coatings in the Ti-B-Si-Al-N system grown by cathodic arc evaporation from TiB2, Ti33Al67, and Ti85Si15 cathodes," J. Vac. Sci. Technol. A 32(6), 061508 (2014).

${ }^{14} \mathrm{~K}$. Jakubka and B. Juttner, "On the influence of surface conditions on initiation and spot types of unipolar arcs in a Tokamak," J. Nucl. Mater. 102, 259-266 (1981).

${ }^{15}$ A. Rogozin and R. Fontana, "Reactive gas-controlled arc process," in Proceedings of XVIIth International Symposium on Discharges and Electrical Insulation in Vacuum (ISDEIV) (IEEE, 1996), Vol. 2, pp. 957-961.

${ }^{16} \mathrm{G}$. Mesyats, "Ecton mechanisms of the vacuum arc cathode spot," IEEE Trans. Plasma Sci. 23(6), 879-883 (1995).

${ }^{17}$ I. Zhirkov, A. Eriksson, A. Petruhins, M. Dahlqvist, A. Ingason, and J. Rosen, "Effect of Ti-Al cathode composition on plasma generation and plasma transport in direct current vacuum arc," J. Appl. Phys. 115, 123301 (2014).

${ }^{18}$ I. Zhirkov, A. Eriksson, and J. Rosen, "Ion velocities in direct current arc plasma generated from compound cathodes," J. Appl. Phys. 114, 213302 (2013).

${ }^{19}$ A. Nikolaev, G. Yushkov, K. Savkin, and E. Oks, "Angular distribution of ions in a vacuum arc plasma with single-slement and composite cathodes," IEEE Trans. Plasma Sci. 41(8), 1923 (2013). 